\title{
Influence of Different Registration Modalities on Navigation Accuracy in Ear, Nose, and Throat Surgery Depending on the Surgical Field
}

Tanja D. Grauvogel, MD; Eric Soteriou; Marc C. Metzger, MD; Ansgar Berlis, MD; Wolfgang Maier, MD

\begin{abstract}
Objectives/Hypothesis: Various invasive and noninvasive registration methods have been established in the past for intraoperative navigation. The present study compared the registration and navigation accuracy of three different registration modalities in anatomical locations of special interest for ear, nose, and throat surgery.

Study Design: Prospective experimental phantom study.

Methods: Four skull models were individually fabricated with a three-dimensional printer based on the patient's computed tomography data sets and fitted with an individual customized silicone skin. Three different registration modalities were examined: 1) invasive marker (IM), 2) oral splint (OS), and 3) laser scan (L). Accuracy measurements were assessed by targeting 26 titanium screws placed over the skull. The overall accuracy and the target registration error for eight selected anatomical locations were measured.

Results: Mean accuracy was $0.67 \pm 0.1 \mathrm{~mm}$ (quadratic mean \pm standard deviation) for IM, $0.98 \pm$ $0.16 \mathrm{~mm}$ for OS, and $1.3 \pm 0.12 \mathrm{~mm}$ for $\mathrm{L}$. The greatest differences in accuracy were found on the mastoid with best accuracy for IM $(0.59 \pm 0.2 \mathrm{~mm} ; P<.05$ vs. $\mathrm{OS}$ and L), followed by OS $(1.23 \pm 0.41 \mathrm{~mm} ; P<.05$ vs. L), and L (1.88 $\pm 0.45 \mathrm{~mm})$. In contrast, only small differences in accuracy were detected in the anterior skull base between the registration modalities
\end{abstract}

From the Department of Otorhinolaryngology-Head and Neck Surgery (т.D.G., E.S., w.M.); and Department of Craniomaxillofacial Surgery (м.c.m.), Albert-Ludwigs-University, Freiburg, Department of Diagnostic Radiology and Neuroradiology, Klinikum Augsburg (А.в.), Germany.

This work was supported by the Faculty of Medicine, Albert-Ludwigs-University, Freiburg, Germany. The authors have no other funding, financial relationships, or conflicts of interest to disclose.

Send correspondence to Tanja D. Grauvogel, MD, Department of Otorhinolaryngology-Head and Neck Surgery, Albert-Ludwigs-University, Killianstrasse 5, 79106 Freiburg, Germany.

E-mail: tanja.grauvogel@uniklinik-freiburg.de
$(\mathrm{IM} 0.75 \pm 0.21 \mathrm{~mm}$, OS $0.71 \pm 0.27 \mathrm{~mm}, \mathrm{~L} 0.93 \pm$ $0.34 \mathrm{~mm})$.

Conclusions: L and OS meet accuracy requirements in the midface and anterior skull base. OS is superior to $\mathrm{L}$ with navigation accuracies comparable to marker registration. However, neither method meets the high precision requirements for lateral skull base surgery.

\section{INTRODUCTION}

Intraoperative navigation is a widespread tool in head and neck surgery. There has been continuous further development since the simultaneous introduction of frameless stereotaxy in Germany, Japan, and the United States in $1986 .{ }^{1}$ Today, various different detection systems based on optical, electromechanical, electromagnetic, or ultrasound measurements are available.

In 2002, the American Academy of OtolaryngologyHead and Neck Surgery published recommendations for the intraoperative use of computer-aided surgery (CAS) in selected cases. Examples of indications in which CAS may be deemed appropriate include: revision sinus surgery; distorted sinus anatomy (of development, postoperative, or traumatic origin); extensive sinonasal polyposis; pathology involving the frontal, posterior ethmoid, and sphenoid sinuses; disease abutting the skull base, orbit, optic nerve or carotid artery; cerebrospinal fluid rhinorrhea or conditions where there is a skull base defect; and benign and malignant sinonasal neoplasm. ${ }^{1}$

In clinical application, the navigation systems reach navigation accuracies with target registration errors (TRE) between 0.5 and $2.77 \mathrm{~mm}$, depending on the registration method and the surgical field, ${ }^{2-4}$ and are considered to be a helpful tool by most of the surgeons. ${ }^{5}$ The use of CAS has proved its worth particularly in primary and revision sinus surgery. ${ }^{5,6}$ 


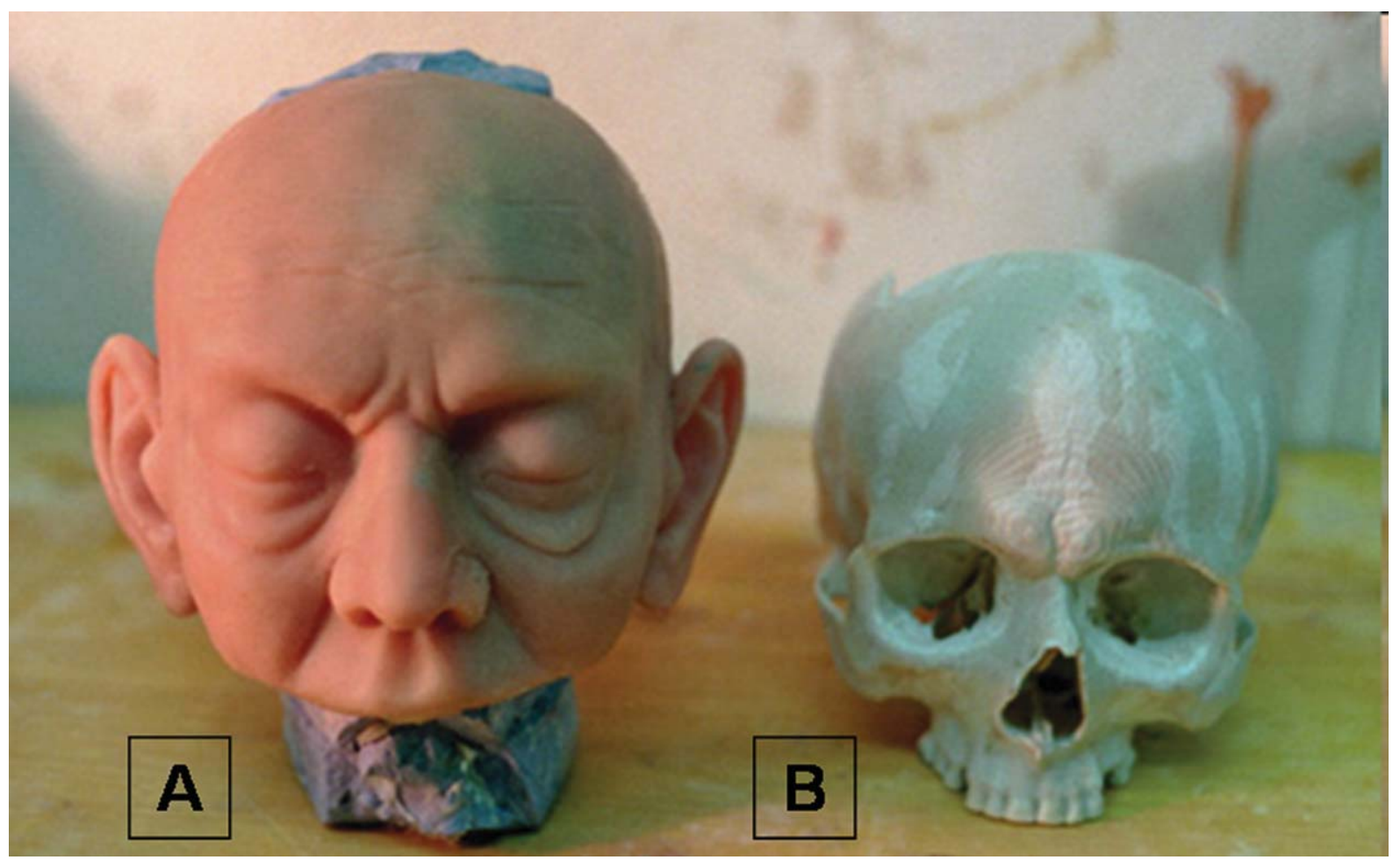

Fig. 1. (A) Individually fabricated silicone skin. (B) Skull model created with a three-dimensional printer based on patient's computed tomography data set. The contours of the silicone skin surface were fabricated fictitious and do not bear any resemblance to real patients.

Problems concerning appropriate referenciation and registration modalities with sufficient navigation accuracy occur when using CAS on the lateral skull base. This is a crucial problem, because maximum accuracy is required in lateral skull base surgery due to complex anatomic structures that are difficult to identify.

Most studies examine only one referenciation or registration modality. ${ }^{7-10}$ Other publications only focus on overall navigation accuracy; differences in accuracy on specific anatomic locations are often not taken into consideration. ${ }^{10,11}$ Therefore, a comparison among different studies often fails because of different experimental set-ups with different navigation systems and measuring methods.

The present study compares the gold standard registration with invasive makers with noninvasive laser surface scanning and noninvasive registration with an oral splint. A skull fixed reference array was used for referenciation. This is the first experimental study that uses different individual skull phantoms based on patients' computed tomography (CT) data sets and that simulates the patients' skin with individual silicone masks.

\section{MATERIALS AND METHODS}

Four individual skull models were fabricated with the help of a three-dimensional printer (Spectrum Z510; Z Corp., Burlington, MA) based on CT scans of real patients. The skull models consisted of a special mixture of powder, binder, and infiltrant for strong, high-definition parts (ZP130/ZB7/Z-Bond 101; Z Corp.). All skulls were fitted with a specially customized silicone skin with an individual surface as used by makeup artists in the theater (Fig. 1). The fabrication of the individual silicone skin was fictitious and unaware of the facial contours of real patients. Finally, all skulls were prepared in the same standardized manner by applying 26 titanium screws (length, 5/ $17 \mathrm{~mm}$; diameter, $1 \mathrm{~mm}$; square cavity, $1 \mathrm{~mm}$; Stryker-Leibinger, Freiburg, Germany) bilaterally distributed over the skull at predetermined anatomical locations. To guarantee precise marker positioning in the anterior and lateral skull base, a part of the cranium was left open during fabrication (Fig. 2). The screws served as target fiducials to determine the TRE. In the setting of invasive fiducial marker registration, four of the 26 titanium screws were defined as registration fiducials. The registration fiducials were located frontoparietal and retroauricular.

Oral silicone splints were fabricated in the dental laboratory of the Department of Craniomaxillofacial Surgery, AlbertLudwigs-University Medical School, Freiburg, Germany. Four titanium screws were placed in alternate order in the oral splint and served as registration fiducials (Fig. 3).

Each of the individually fabricated skull models underwent CT scan (Somatom Sensation 16; Siemens AG, Munich, Germany) with a slice thickness of $1 \mathrm{~mm}$, gantry $0^{\circ}$, resolution $(\mathrm{X} \times \mathrm{Y}) 512 \times 512$, and pixel size $(\mathrm{mm}) 0.396 \times 0.396$. The DICOM data were imported into the navigation software (PatXfer 5.2; BrainLAB AG, Feldkirchen, Germany) and all titanium screws were marked in the coronal, axial, and sagittal slices with a special software program (iPlan Cranial 2.5; BrainLAB AG) (Fig. 4). Vector Vision (BrainLAB AG) was used as the navigation system.

Navigation measurements were made in the operating room by positioning the skull in a rubber ring on the operating table in a standardized manner. The skull reference array (SRA) was screwed into the skull closely behind the frontal hairline, comparable to clinical conditions (Fig. 2, Fig. 3).

The registration procedure was repeated five times per skull. After each registration, each target fiducial was actuated 


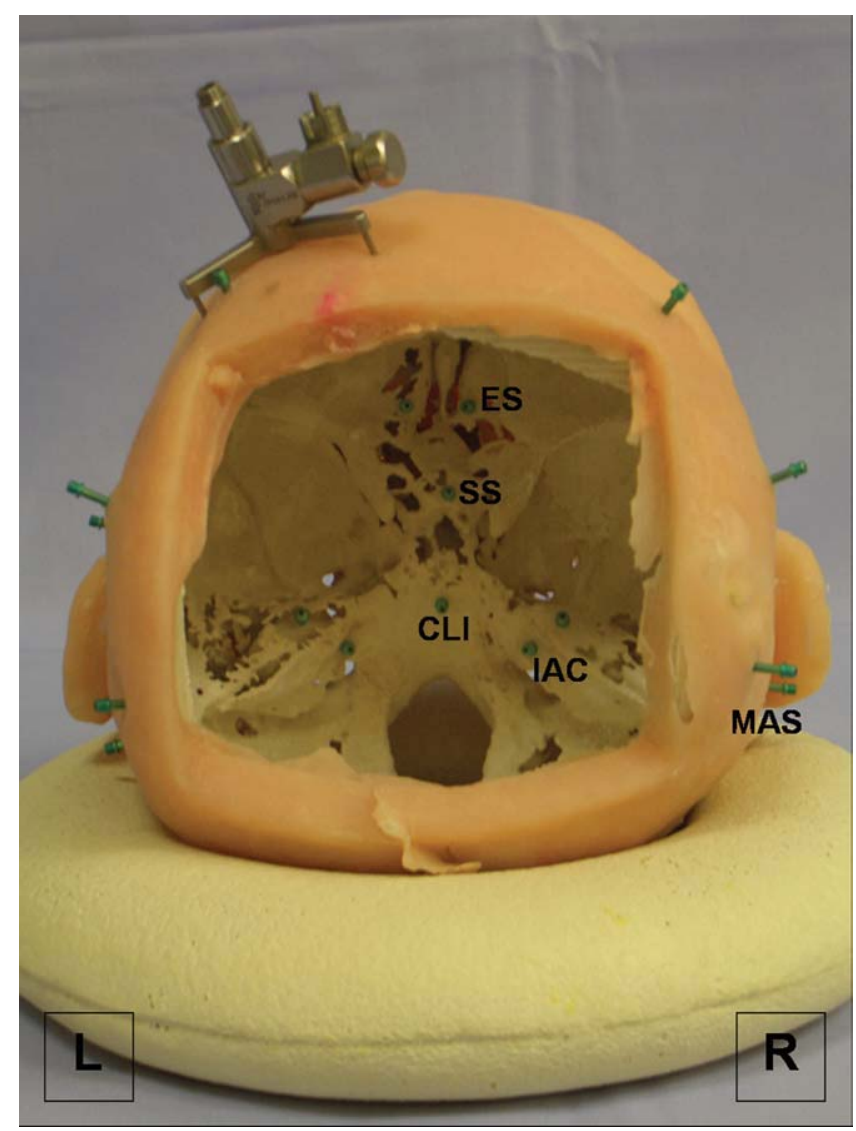

Fig. 2. Skull model with an opening in the calvarium, skull reference array fixation, and titanium screws drilled in the skull and skull base. Sites of target fiducials are marked with labels. ES = ethmoid sinus, $S S=$ sphenoid sinus, $C L I=$ clivus, $I A C=$ internal auditory canal, MAS $=$ mastoid.

five times with the navigation pointer. The experiments were carried out by two independent operators.

Three different registration modalities were compared in the study: 1) the registration with invasive fiducials (skull reference array/invasive marker, SRA/IM), which was defined as the gold standard; 2) oral splint registration (skull reference array/oral splint, SRA/OS) (Fig. 3); and 3) laser surface registration (skull reference array/laser, SRA/L) (Fig. 5).

Measurements were taken by placing the navigation pointer on the target fiducial in the squared cavity of each titanium screw (Fig. 5). With regard to target fiducials affixed to the skull base (ethmoid sinus, sphenoid sinus, clivus, internal auditory canal), exact positioning of the pointer was guaranteed by an aperture in the calvarium. The virtual and the real coordinates of each fiducial ( $\mathrm{x}, \mathrm{y}, \mathrm{z}$ coordinates) were displayed by the navigation system. The Euclidian distance could then be calculated for each target fiducial, which is consistent with the TRE $\mathrm{d}(\mathrm{x}, \mathrm{y}, \mathrm{z})=\mathrm{d}(\mathrm{x}, \mathrm{y}, \mathrm{z})=\sqrt{\Delta x^{2}+\Delta y^{2}+\Delta z^{2}}$.

Data are expressed as quadratic mean values $(\mathrm{mm}) \pm$ standard deviation $(\mathrm{mm})$. Statistical analysis was performed with a Tukey test and variance analysis. A probability $P<.05$ was considered statistically significant.

\section{RESULTS}

The mean accuracy (with respect to all 26 titanium screws) was $0.67 \pm 0.11 \mathrm{~mm}$ (quadratic mean \pm SD) for registration with invasive markers, $0.98 \pm 0.16 \mathrm{~mm}$ for oral splint registration, and $1.3 \pm 0.12 \mathrm{~mm}$ for laser surface scanning (Fig. 6). The mean TRE of pair-point registration $(\mathrm{PPR})$ with invasive marker registration was significantly lower than the mean TRE of PPR with oral splint $(P<.05)$, and laser surface scanning $(P<.05)$ (Fig. 6). A statistically significant difference of mean accuracy could also be detected between oral splint registration and registration by laser surface scanning $(0.98 \pm 0.16 \mathrm{~mm}$ vs. $1.3 \pm 0.12 \mathrm{~mm}, P<.05)$ (Fig. 6$)$.

TREs on selected locations of special interest for the ear, nose, and throat (ENT) surgeon are given in Figure 7. Mean values for invasive marker registration showed the lowest TRE in nearly all locations, but no statistically significant differences could be found between the three registration methods in the anterior skull base (frontal sinus and ethmoid sinus) (Fig. 7).

In all other locations, invasive marker registration was found to be significantly superior to laser surface registration $(P<.05)$ but not to oral splint registration with the exception of the mastoid. On the one hand, the mastoid was the location with the biggest differences in mean accuracy $(0.59 \pm 0.20$ vs. $1.23 \pm 0.41$ vs. $1.88 \pm$ $0.45 \mathrm{~mm}$ ), and on the other hand, the only location at which the accuracy of invasive marker registration was significantly better than the oral splint registration $(P<$ .05) (Fig. 7)

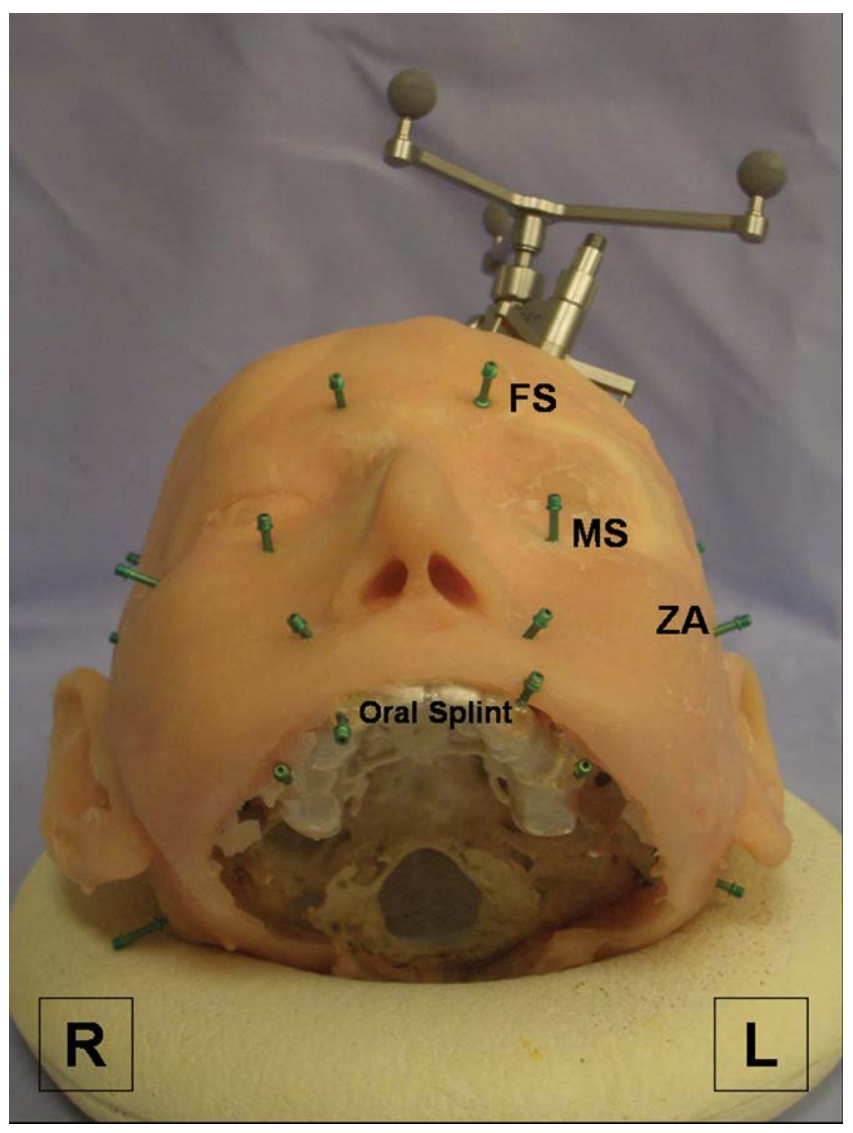

Fig. 3. Skull model with fixed skull reference array and oral splint. The four titanium screws in the oral splint served as registration fiducials. Sites of target fiducials are marked with labels. FS = frontal sinus; $M S=$ maxillary sinus; $Z A=$ zygomatic arch. 


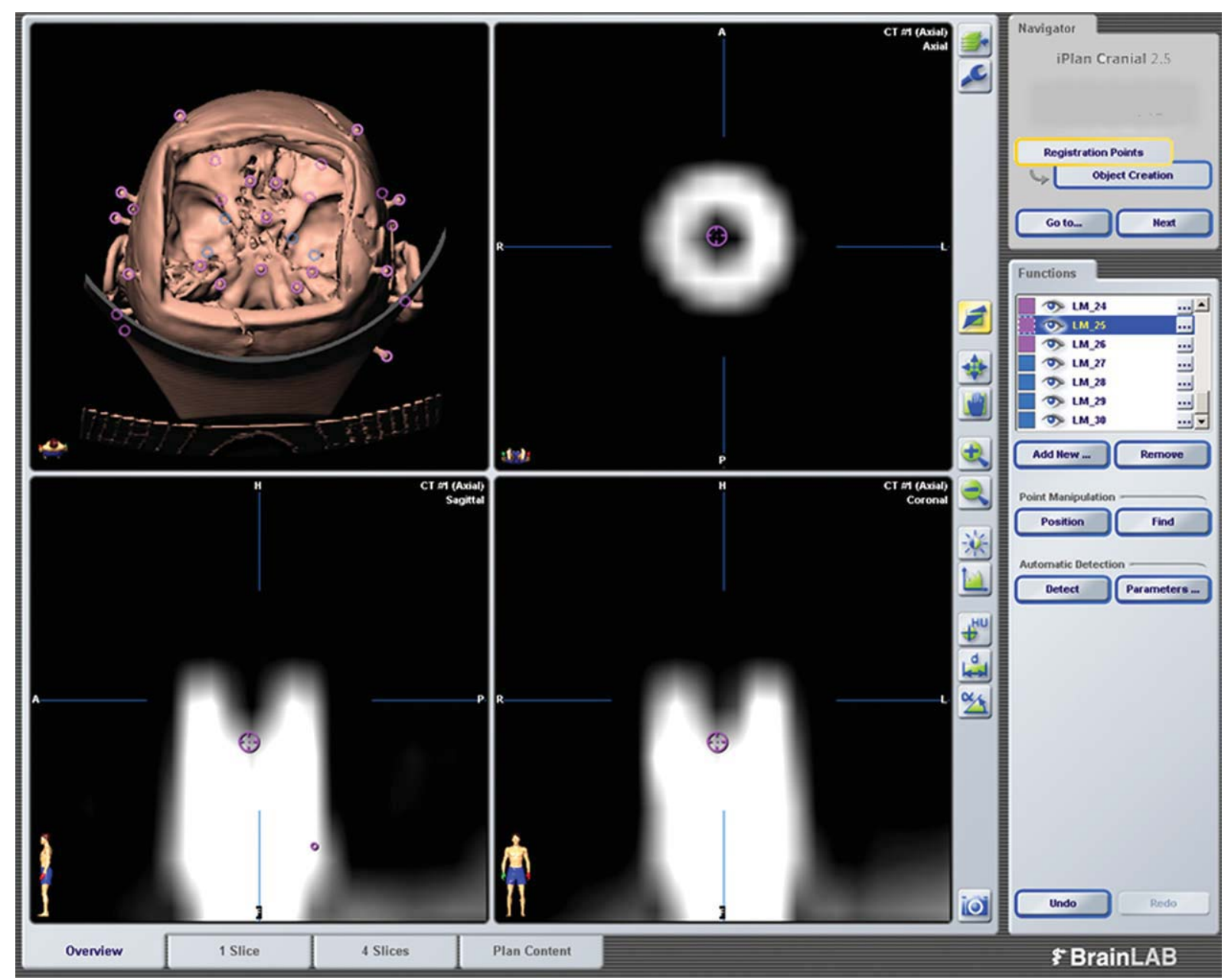

Fig. 4. Marked titanium screws in the computed tomography scan via BrainLAB navigation software (iPlan Cranial 2.5, BrainLAB AG, Feldkirchen, Germany).

Accuracy of laser surface scan registration showed the highest TRE in most locations. Compared to oral splint registration, laser surface scan registration was statistically significantly worse on maxillary sinus, sphenoid sinus, clivus, and mastoid $(P<.05)$ (Fig. 7).

\section{DISCUSSION}

Regardless of the diversity of referenciation and registration modalities, a navigation system has to meet certain demands. It should be of assistance to the surgeon and increase efficacy and safety. ${ }^{1,5}$ In ENT surgery a frameless navigation system that allows unimpeded head movement is necessary. The use of a navigation system helps to avoid wide-open surgery and facilitates minimally invasive procedures. Therefore, high system accuracy in the 1.0 to $2.0 \mathrm{~mm}$ range is mandatory for rhinologic procedures. ${ }^{12}$ In the clinical setting, the achievable TRE ranges from 0.5 to $2.77 \mathrm{~mm} .^{2-5}$ In lateral skull base surgery, navigation accuracy should be even $<1 \mathrm{~mm}$ due to its complex anatomic structure, ${ }^{13}$ particularly in some high-precision surgery, for example, cochlear implant surgery in malformed temporal bone, where an accuracy of $0.5 \mathrm{~mm}$ or less is desirable.
Clinical studies have shown that available navigation systems meet most of these requirements, but there is considerable variation in navigation accuracy depending on different anatomic regions and different registration modalities. ${ }^{3,4}$

From this point of view, it is important to show what type of navigation system and registration method is sufficient in which regions, and where an improvement of navigation technique is required. The present study compared the overall accuracy and the accuracy in different surgical fields using three different registration modalities.

For the first time, investigations were made with the help of individually fabricated skull models based on CT scans of real patients. The use of cadavers could be avoided, and unlike industrially manufactured skull models, which were used in other studies, ${ }^{1,7}$ our custommade skulls differed from each other in size and anatomy, comparable to human beings.

Although skull fabrication requires time and money, the models are durable and can be used at any time. Additionally, the skull models were provided with an individual silicone mask for skin simulation. The skulls had a realistic facial outline with nose and cheek, which 


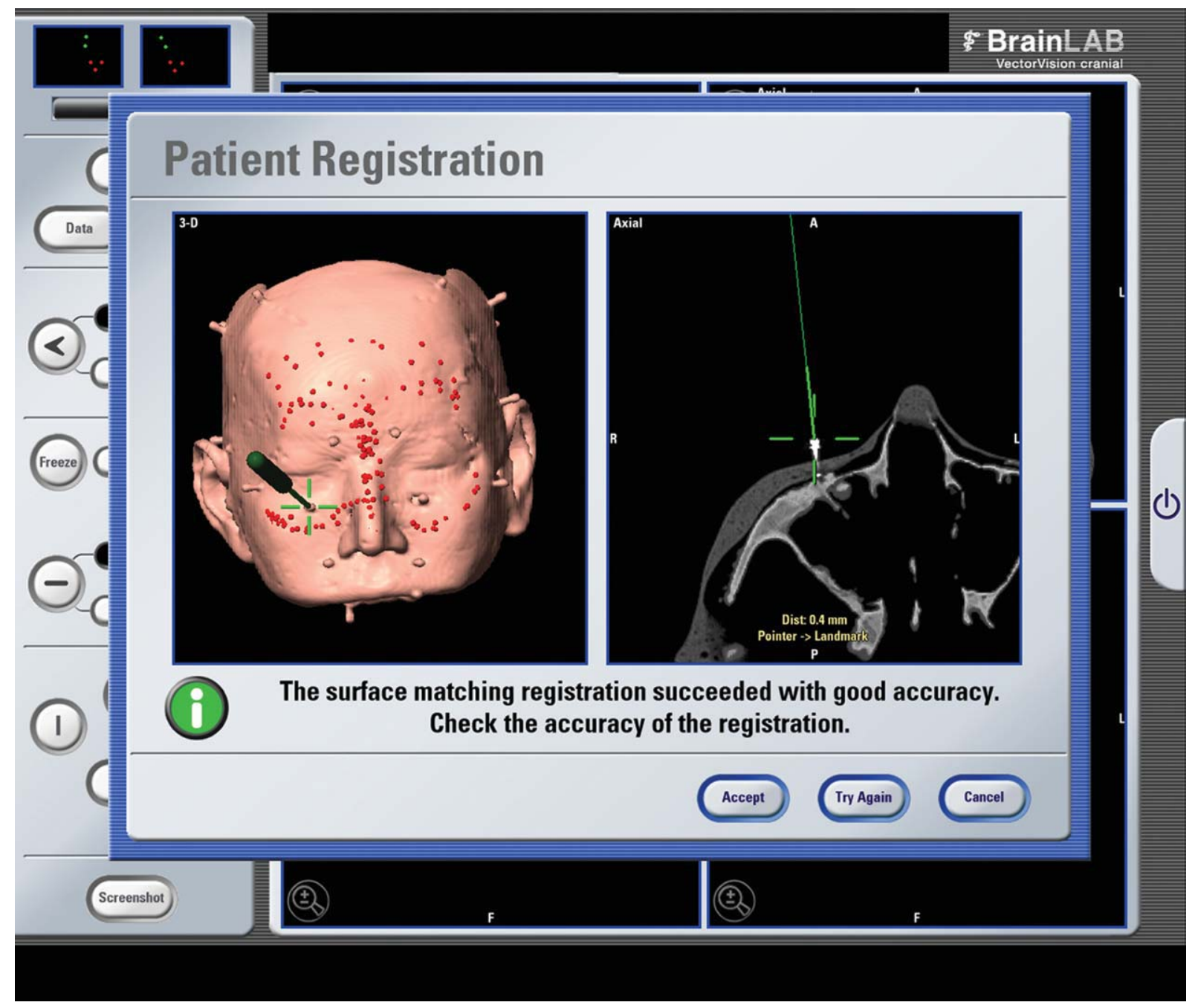

Fig. 5. Screenshot after laser surface registration with the Vector Vision navigation system (BrainLAB AG, Feldkirchen, Germany). Control of accuracy is by actuating a titanium screw with the pointer.

is of special interest for laser surface registration. With this setup, we achieved a registration situation that is very close to the real procedure and the intraoperative circumstances during patient registration. However, the influence on navigation accuracy of skin surface altera-

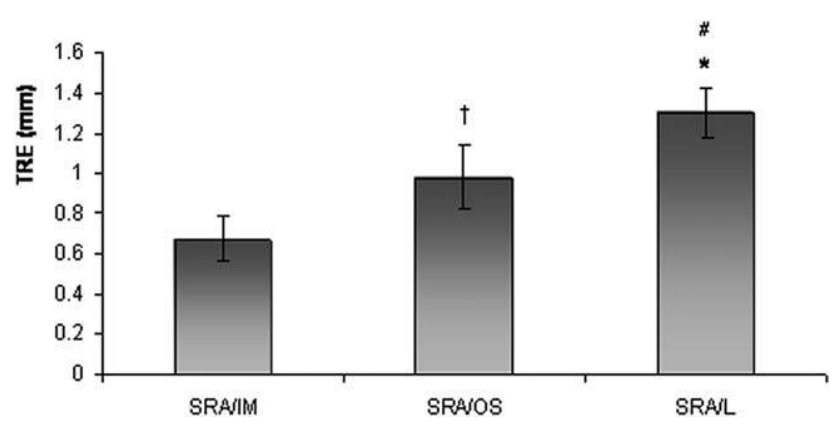

Fig. 6. Target registration error (TRE), mean accuracy with respect to all 26 titanium screws: quadratic mean \pm standard deviation in mm; †SRA/OS vs. SRA/IM; *SRA/L vs. SRA/IM; \#SRA/L vs. SRA/ OS; $P<.05$; Tukey test. SRA $=$ skull reference array; IM = invasive marker; $\mathrm{L}=$ laser scan. tions caused by edema, tumor, or skin turgor could not be imitated by this method.

Currently available registration modalities include PPR with invasive (titanium screws) and noninvasive

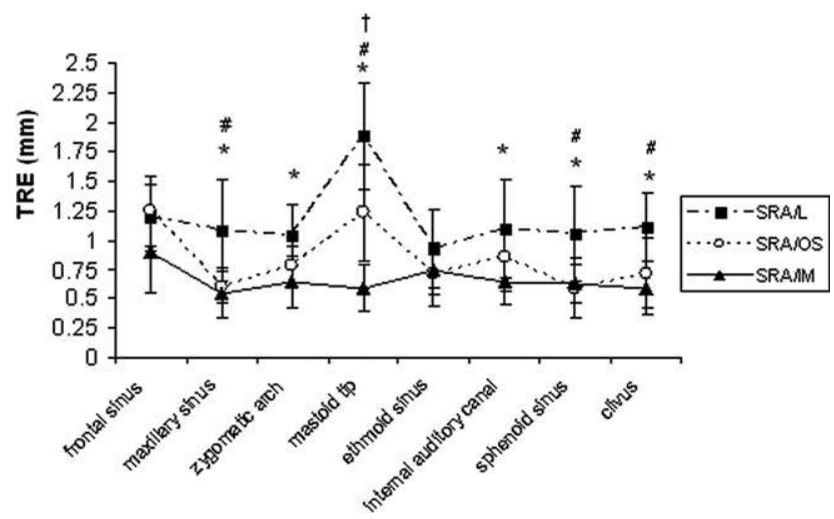

Fig. 7. Target registration error (TRE) on selected locations: quadratic mean \pm standard deviation in $\mathrm{mm}$; †SRA/OS vs. SRA/IM; ${ }^{*} \mathrm{SRA} / \mathrm{L}$ vs. SRA/IM; \#SRA/L vs. SRA/OS; $P<.05$; Tukey test. SRA $=$ skull reference array; $\mathrm{IM}=$ invasive marker; $\mathrm{L}=$ laser scan. 
markers (skin affixed markers, anatomic landmarks), registration with the help of dental splints, or laser surface scanning.

In the present study, invasive titanium screws were used for registration because this represents the gold standard of pair-point registration yielding the highest navigation accuracy. ${ }^{6,10}$ Registration with anatomic landmarks has already been investigated in several studies showing that registration with bony landmarks generates results that are less exact in comparison with other noninvasive registration techniques. ${ }^{5,14}$ Therefore, in the present study we deliberately focused on localizationspecific exactness of the most reliable noninvasive registration methods in targets of lateral and frontal skull base, and decided to include oral splint and laser registration (which are both routinely available at our institution) into our study, but not registration by anatomic landmarks.

\section{Invasive Marker Registration}

Registration with invasive fiducials offers maximum accuracy and is still regarded as the gold standard. ${ }^{6,10}$ The titanium screws utilized are easily detectable on CT scan and on the patient. Their application is independent of patient's dentition or of surface alteration due to trauma, swelling, or tumor. However, the screws must be placed before the CT scan, which includes discomfort for the patient and might require a second scan in some cases.

Because titanium screws offer the possibility of exact and rigid positioning and can be precisely actuated with the navigation pointer, we also used them as target fiducials for accuracy measurements in our study. The cavity in the middle of the screw minimized measurement errors due to the possibility of exact fiducial marking in the navigation software and during screw actuation with the navigation pointer.

We took four titanium screws for registration and placed them bilaterally in retroauricular and parietal position in the hairline and obtained high overall accuracy of $0.67 \mathrm{~mm}$ comparable to the results of Luebbers et al. ${ }^{15}$ Other experimental studies using invasive marker registration reported registration accuracies with different navigation systems between 1.00 and $1.34 \mathrm{~mm}^{10}$ and 0.3 and $1.8 \mathrm{~mm} .{ }^{11}$ All authors reported higher navigation accuracy with invasive marker registration compared to noninvasive anterior registration methods. Differences in overall accuracy among the studies might be explained by different number and arrangement of fiducials. In contrast to the studies cited above, in which invasive fiducials were placed in the midface, we focused on marker localizations that are cosmetically tolerable (behind the ear and behind the hairline), and therefore could be used in selected clinical cases where a maximum of navigation accuracy is mandatory. The fiducial arrangement and number was in accordance with the guidelines published by West and coworkers in $2001 .^{16}$ The high accuracy of $0.56 \mathrm{~mm}$ at the mastoid in our study might be due to the proximity of the target fidu- cial on the mastoid to the registration fiducial positioned retroauricular.

\section{Oral Splint Registration}

An alternative noninvasive registration tool is the oral splint. Splints are mainly used in the field of oral and craniomaxillofacial surgery. They can be easily applied in a toothed maxilla and rapidly registered. $^{7,10,17}$ If reregistration is necessary, the splint is available at any time..$^{7,10,17} \mathrm{~A}$ dental laboratory is necessary for splint fabrication. Moreover, a second CT scan may be necessary in some cases, because the preoperative scan must be taken with the splint. There are two different types of oral splints. One type affixes only four to five registration fiducials. ${ }^{7,10,18}$ The other type, the so-called dental bite block, is usually provided with a greater number of registration fiducials and additionally affixes a reference star with the help of a frame. ${ }^{6,8}$

In the present study we used an oral splint containing four alternating titanium screws. As previously reported, the splint is easy to handle and not prone to impairment, even in multiple registrations with removal and repositioning of the splint. ${ }^{7,18}$

We measured a relatively high overall accuracy of $0.98 \mathrm{~mm}$. Comparable average TREs are reported by Schramm et al. ${ }^{18}$ Luebbers et al. differentiated between periorbital, viscerocranium, and neurocranium regions with the best average TRE periorbitally of $1.1 \mathrm{~mm}$, followed by $1.3 \mathrm{~mm}$ at the viscerocranium, and $2.3 \mathrm{~mm}$ at the neurocranium. ${ }^{15}$ In this study, 170 drilled holes with a diameter of $>1 \mathrm{~mm}$ served as target fiducials and were distributed over the skull for regional precision assessment; specific anatomic locations were not examined.

Specific measurements on the lateral skull base are rare and show large accuracy variation, with a range between 0.73 and $3.31 \mathrm{~mm}^{6-8}$ The large differences in accuracy probably are due to different experimental or clinical settings, distribution of the registration fiducials in the oral splint, and the position of the splint in relation to the reference system.

Based on our results, registration with an oral splint has to be considered as a reliable and reasonable alternative registration method to invasive marker registration even in many skull base procedures, whereby best possible fiducial placement is necessary. The oral splint may be sufficient even in the lateral skull base, but this depends on the procedure in question. In the temporomastoidal region, the accuracy of oral splint registration is comparatively low and does not meet high precision demands, for example, for cochlear implant surgery.

In the meantime, attempts are being made to facilitate oral splint fabrication by testing different materials. ${ }^{17}$ As a consequence, oral splints would become cheaper and fabrication could be done by the surgeon without time delay, which would facilitate routine application in ENT surgery. 


\section{Laser Surface Registration}

Laser surface registration is nowadays a widespread tool especially in ENT surgery. It is a noninvasive registration method that uses the anatomy of the patient's head and face by scanning it with a laser beam. Therefore, the need for second planning imaging can be avoided.

The currently available systems are the Fazer, which acquires 300 points during a surface scan (StealthStation Treon; Medtronic, Inc. Minneapolis, MN) and the Z-Touch (BrainLAB AG, Feldkirchen, Germany) (200 points), which was used in our study. The producers of these products recommend scanning the skin of the midface to get best accuracy results.

Instead of periorbital and frontal registration areas, Marmulla et al. tested automated laser registration of the auricle with the aim of improving navigation accuracy in lateral skull base surgery. With this method, a mean TRE of $0.9+0.3 \mathrm{~mm}$ periauricular was attained as long as the auricle was not deformed during CT imaging or laser scanning. ${ }^{19}$ Laser surface scanning with the Fazer reached accuracies about $1.8 \mathrm{~mm}$ in cadaver studies. ${ }^{11}$

The mean accuracy value obtained by laser surface registration in our study is comparable to accuracies measured in previous experimental studies using the same navigation system. ${ }^{9,15}$ In the clinical setting mean accuracies with the Z-Touch of $2.4 \mathrm{~mm}^{3}$ and $2.77 \mathrm{~mm}^{4}$ have been reported. Accuracy on the basis of anatomic landmarks cannot be as precise as on the basis of screws, because the vast majority of landmarks are not exactly defined.

With regard to special anatomical locations in the field of ENT, the few studies that exist mainly performed accuracy measurements by actuating anatomical landmarks. Raabe et al. reported values between 5.0 and 1.9 $\mathrm{mm}$ on the nasion and between 10.3 and 0.8 on the external auditory canal. ${ }^{3}$ Schlaier et al. reported TRE of $2.61 \pm 2.06$ (distance to tragus), $2.19 \pm 1.98$ (distance to lateral canthus) and $2.77 \pm 1.64$ to any target fiducial. ${ }^{4}$ The impreciseness of measurements on anatomical landmarks is due to imprecise actuation and is expressed in high standard deviations.

Our measurements on different anatomical locations are mainly consistent with those measured by Ledderose and coworkers. ${ }^{9}$ The better results at the internal auditory canal ( $1.09 \mathrm{~mm}$ vs. 1.76 petrous bone) are probably due to better actuation of the titanium screw through the opening of the calvarium in our study. We think that overall additional factors, such as, for example, position of the head to the reference star or position of the reference star to the camera, might influence measurement results and explain different values. These influencing variables were already discussed by Schlaier et al. in the clinical setting. ${ }^{4}$

\section{CONCLUSION}

The aim of modern CAS must be the use of noninvasive registration methods. Laser surface scanning and oral splint registration, which have the advantage of being noninvasive, show acceptable accuracies in the midface and anterior skull base, but do not meet high precision requirements in distinct regions of the lateral skull base. Laser surface scanning is a noninvasive registration alternative for sinus surgery, but possible loss in accuracy has to be considered due to skin surface alterations. Oral splint registration is very accurate in the midface and anterior skull base, comparable to invasive marker registration, but beyond its weakness on the lateral skull, it cannot be applied in toothless patients. So, an alternative noninvasive method for high-precision registration, especially for lateral skull base surgery, should be developed.

\section{Acknowledgments}

The authors thank Professor Dr. J. Schulte-Moenting from the Institute of Biostatistics and Medical Informatics, University of Freiburg, Germany for his support in statistical analysis, and Ms I. Neu, makeup artist at the theater of Freiburg, Germany for fabricating the silicone masks.

\section{BIBLIOGRAPHY}

1. Wise SK, DelGaudio JM. Computer-aided surgery of the paranasal sinuses and skull base. Expert Rev Med Devices 2005;2:395-408.

2. Caversaccio M, Nolte LP, Hausler R. Present state and future perspectives of computer aided surgery in the field of ENT and skull base. Acta Otorhinolaryngol Belg 2002; 56:51-59.

3. Raabe A, Krishnan R, Wolff R, Hermann E, Zimmermann $M$, Seifert V. Laser surface scanning for patient registration in intracranial image-guided surgery. Neurosurgery 2002;50:797-801, discussion 802-793.

4. Schlaier J, Warnat J, Brawanski A. Registration accuracy and practicability of laser-directed surface matching. Comput Aided Surg 2002;7:284-290.

5. Citardi MJ, Batra PS. Intraoperative surgical navigation for endoscopic sinus surgery: rationale and indications. Curr Opin Otolaryngol Head Neck Surg 2007;15:23-27.

6. Balachandran R, Fitzpatrick JM, Labadie RF. Accuracy of image-guided surgical systems at the lateral skull base as clinically assessed using bone-anchored hearing aid posts as surgical targets. Otol Neurotol 2008;29:10501055.

7. Eggers G, Muhling J. Template-based registration for image-guided skull base surgery. Otolaryngol Head Neck Surg 2007;136:907-913.

8. Labadie RF, Shah RJ, Harris SS, et al. In vitro assessment of image-guided otologic surgery: submillimeter accuracy within the region of the temporal bone. Otolaryngol Head Neck Surg 2005;132:435-442.

9. Ledderose GJ, Stelter K, Leunig A, Hagedorn H. Surface laser registration in ENT-surgery: accuracy in the paranasal sinuses-a cadaveric study. Rhinology 2007;45: 281-285.

10. Metzger MC, Rafii A, Holhweg-Majert B, Pham AM, Strong B. Comparison of 4 registration strategies for computeraided maxillofacial surgery. Otolaryngol Head Neck Surg 2007;137:93-99.

11. Schicho K, Figl M, Seemann R, et al. Comparison of laser surface scanning and fiducial marker-based registration in frameless stereotaxy. Technical note. $J$ Neurosurg 2007;106:704-709.

12. Cartellieri M, Vorbeck F. Endoscopic sinus surgery using intraoperative computed tomography imaging for updating a three-dimensional navigation system. Laryngoscope 2000;110:292-296. 
13. Caversaccio M, Zulliger D, Bachler R, Nolte LP, Hausler R. Practical aspects for optimal registration (matching) on the lateral skull base with an optical frameless computeraided pointer system. Am J Otol 2000;21:863-870.

14. Mascott CR, Sol JC, Bousquet P, Lagarrigue J, Lazorthes Y, Lauwers-Cances V. Quantification of true in vivo (application) accuracy in cranial image-guided surgery: influence of mode of patient registration. Neurosurgery 2006; 59:ONS146-ONS156, discussion ONS146-ONS156.

15. Luebbers HT, Messmer P, Obwegeser JA, et al. Comparison of different registration methods for surgical navigation in cranio-maxillofacial surgery. J Craniomaxillofac Surg 2008;36:109-116.

16. West JB, Fitzpatrick JM, Toms SA, Maurer CR Jr, Maciunas RJ. Fiducial point placement and the accuracy of point-based, rigid body registration. Neurosurgery 2001; 48:810-816, discussion 816-817.

17. Strauss G, Spitzer C, Dittrich E, Hofer M, Strauss M, Luth T. Modified procedure for patient registration for navigation control instruments in ENT surgery [in German]. HNO 2009;57:153-159.

18. Schramm A, Gellrich NC, Schimming R, Schon R, Gutwald $R$, Schmelzeisen R. Noninvasive registration in computer assisted cranio-maxillofacial surgery. In: Worn $\mathrm{H}$, Muhling J, Vahl C, Meinzer HP, eds. Rechner- und sensorgestützte Chirurgie. Bonn, Germany: Kollen, 2002: 258-269.

19. Marmulla R, Eggers G, Muhling J. Laser surface registration for lateral skull base surgery. Minim Invasive Neurosurg 2005;48:181-185. 